\title{
Die Zusammensetzung der Nucleinsäuren aus Thymus und aus Heringsmilch. \\ Von
}

H. Stendel.

(Aus dem physiologischen Institut in Heidelberg.)

(Der Redaktion zugegangen am 6. Oktober 1906.)

Einer restlosen quantitativen Aufspaltung der Nucleinsäuren $\left.{ }^{1}\right)$ standen bisher große Schwierigkeiten gegenüber; von denen das Auswaschen der massenhaften Barytniederschläge, die bei der Neutralisierung der Schwefelsäure und Phosphorsäure und zum zweiten Male bei der Zersetzung der Phosphorwolframsäurefällung entstanden, nicht die geringsten waren. Es bestand also gar kein Zweifel, daß die zum Schluß erhaltenen Zahlen für die Ausbeuten an Spaltungsprodukten, besonders an Basen, nur Mindestzahlen waren und nicht den wirklichen Betrag anzeigten, wie er im Molekül der Nucleinsäure enthalten war. Dazu kam ferner, daß das lange Sieden mit Mineralsäuren nicht allein eine hydrolysierende, sondern auch eine oxydierende Wirkung haben konnte, sodaß man neben primären Spaltungsprodukten zum Schluß auch solche fand, die aus diesen erst sekundär durch Oxydation hervorgegangen sein konnten. Neben Guanin ließ sich regelmäßig eine kleine Menge Xanthin isolieren, neben Adenin Hypoxanthin und neben Cytosin Uracil.

Diese Schwierigkeiten haben sich jetzt, wenigstens für die Alloxurbasen, umgehen lassen, nachdem ich die Wirkung starker Salpetersäure ${ }^{2}$ ) auf Nucleinsäure kennen gelernt hatte. Man erhält auf diesem Wege sofort die Nitrate des Guanins und Adenins fast quantitativ, der geringe Teil, der noch in Lösung bleibt, kann vernachlässigt werden, desgleichen die minimalen Mengen von Hypoxanthin und Xanthin, die bei der Oxydation entstehen. Man kann diese Fehler auf ein Minimum S. 332.

1) Diese Zeitschrift, Bd. XLII, S. 165; Bd. XLIII, S. 402; Bd. XLVI,

2) Diese Zeitschrift, Bd. XLVIII, S. 425. 
herabdrücken, wenn man bei niedriger Temperatur arbeitet und nachher die Flüssigkeit mindestens 8-14 Tage zur Krystallisation sich selbst überläßt. Ich habe bisher 5 Versuche dieser Art, jedesmal an $100 \mathrm{~g}$ lufttrockenem nucleinsauren Kupfer angestellt, ${ }^{1}$ ) nach 2-3 wöchentlichem Stehen: die Nitrate abgesaugt, kurz mit verdünnter Salpetersäure, dann mit Alkokol und Äther nachgespült und jedesmal die Menge der Nitrate bestimmt. Ich erhielt fast genau die gleichen Zahlen in sämtlichen 5 Versuchen: 17,5-18,5 g. Aus diesen ließen sich nun nach bekannten Methoden isolieren:

$5,3 \mathrm{~g}$ Guanin und 6,28 $\mathrm{g}$ Adenin, entsprechend einer Stickstoffmenge von $2,461 \mathrm{~g}$ resp. $3,266 \mathrm{~g}$.

Rechnet man diese Zahlen in Prozente des Gesamtstickstoffs um, so ergibt sich folgendes:

Das von mir benutzte nucleinsaure Kupfer war lufttrocken und enthielt nach mehreren Kjeldahl-Bestimmungen rund $8,5 \% \mathrm{~N}$.

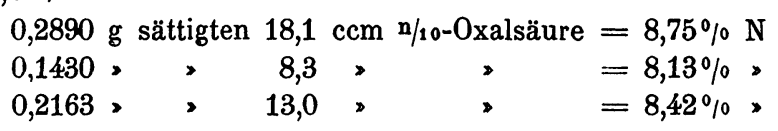

Es würden also, in Prozenten des Gesamtstickstoffs: ausgedrückt, auf Guanin 28,95\% und auf Adenin 38,42\% entfallen. Damit wäre schon über die Hälfte des Stickstoffs: der Nucleinsäure aufgelöst. Der noch verbleibende Rest besteht. sicher zum Teil aus Thyminstickstoff, denn Thymin konnte von mir auch bei der Spaltung mit Salpetersäure isoliert werden, zum anderen ist Cytosin im Molekül der Nucleinsäure gefunden. Dieses Cytosin tritt aber bei der Oxydation mit Salpetersäure als Uracil auf, sodaß hier die Betrachtung über die Verteilung des Stickstoffs an den Produkten der Salpetersäurespaltung nicht weiter fortgesetzt werden kann; man muß, und das wird hier erlaubt sein, auf die Resultate zurückgreifen, die bei der Schwefelsäurespaltung erhalten worden sind, und muß dabei nur das eingangs Gesagte im Auge behalten, daß sicher ein Teil des Cytosins bei der Darstellung

1) Der Weg war der gleiche, den ich Bd. XLVIII, S. 425 veröffentlicht habe.

Hoppe-Seyler's Zeitschrift f. physiol. Chemie. XLIX. 
verloren, ein anderer schon vorher während der Spaltung zu Uracil oxydiert worden ist. Nimmt man unter dieser Voraussetzung die Zahlen für Cytosin und Thymin aus der Schwefelsäurespaltung zu Hilfe, ${ }^{1}$ ) so erhält man folgende Werte in Prozenten des Gesamtstickstoffs:

\begin{tabular}{ll} 
Guanin & $28,95 \%$ \\
Adenin & $38,42 \%$ \\
Cytosin & $\left.11,47 \% \%^{1}\right)$ \\
Thymin & $\left.13,11 \%^{1}\right)$ \\
\cline { 2 - 3 } & $\left.91,95 \% \%^{2}\right)$
\end{tabular}

Es besteht also offenbar der stickstoffhaltige Teil des Moleküls der Nucleinsäure lediglich aus diesen 4 Körpern; nun ist bisher aber die Formel der Nucleinsäure nur für 14 $\mathrm{N}$-Atome berechnet worden und es folgt jetzt mit Notwendigkeit, daß man sie auf $15 \mathrm{~N}$-Atome bringen muß, um sämtliche Spaltungsprodukte in ihr unterzubringen. Schon bei meinen Analysen des Kupfersalzes der Nucleinsäure ${ }^{3}$ ) war mir aufgefallen, daß meine Stickstoffwerte für die alte Formel sämtlich zu hoch ausgefallen waren; diese Schwierigkeit fällt gleichfalls fort, wenn man mit 15 Stickstoffatomen rechnet. Es berechnet sich nämlich

$$
\begin{aligned}
& \text { für } \mathrm{C}_{40} \mathrm{H}_{53} \mathrm{Cu}_{2} \mathrm{~N}_{15} \mathrm{O}_{28} \mathrm{P}_{4}: \quad \text { Gefunden: }{ }^{4} \text { ) Alte Formel: } \\
& \mathrm{Cu}=9,01 \% \quad 9,77 \%, 9,07 \% \quad \mathrm{Cu}=9,10 \% \\
& \mathrm{P}=8,78 \% \quad 8,87 \%, 8,79 \% \quad \mathrm{P}=8,89 \% \\
& \mathrm{~N}=14,92 \% \quad 14,37 \%, 14,47 \%, 14,48 \% \text { (Kjeldahl) } \mathrm{N}=14,10 \%
\end{aligned}
$$
$\mathrm{zu}$ der nunmehr verbesserten Formel besser als zu der früher gebräuchlichen.

1) Diese Zeitschrift, Bd. XLIII, S. 403 ; Bd. XLVI, S. 334. Die Nucleinsäuren aus Thymus und aus Heringsmilch einander gleichzusetzen, ist erlaubt, weil ich in drei bisher nicht veröffentlichten quantitativen Spaltungsversuchen der Nucleinsäure aus Heringsmilch mit siedender Schwefelsäure im wesentlichen dieselben Zahlen erhalten habe wie bei der Nucleinsäure aus Kalbsthymus.

2) Das Ammoniak, das bei der Spaltung mit siedender Schwefelsäure auftritt, läßt sich ungezwungen ableiten aus der sekundären Oxydation von Guanin, Adenin und Cytosin zu Xanthin, Hypoxanthin und Uracil.

3) Diese Zeitschrift, Bd. XLVI, S. 335.

4) Diese Zeitschrift, Bd. XLVI, S. 335 . 
Sollen aus der Nucleinsäure nun je ein Molekül Guanin, Adenin, Cytosin und Thymin hervorgehen, so würde sich für $\mathrm{C}_{40} \mathrm{H}_{53} \mathrm{Cu}_{2} \mathrm{~N}_{15} \mathrm{O}_{26} \mathrm{P}_{4}$ berechnen:

$$
\begin{aligned}
& \text { Verlangt: Guanin } 10,72 \% \\
& \text { Adenin } 9,58 \% \\
& \text { Cytosin } 7,86 \% \\
& \text { Thymin 8,93\% }
\end{aligned}
$$

Experimentell ist dazu von mir folgendes gefunden worden: Die $100 \mathrm{~g}$ lufttrockenes nucleinsaures Kupfer mit einem Stickstoffgehalt von $8,5 \% \mathrm{~N}$ entsprechen $58,82 \mathrm{~g}$ vom trockenen Salze, und für dieses ergeben sich an Ausbeuten:

$$
\begin{array}{cl}
\text { Gefunden: } & \text { Guanin } 9,01 \% \\
& \text { Adenin } 10,68 \% \\
& \text { Cytosin } \left.4,26 \%{ }^{1}\right) \\
& \text { Thymin } 8,33 \% \text { ) }
\end{array}
$$

Die Übereinstimmung ist besser, wie man sie eigentlich bei solchen komplizierten Untersuchungen erwartet, und die Zahlen sprechen eine deutliche Sprache: Die 4 stickstoffhaltigen Komponenten der Nucleinsäure und nur diese vier kommen im molekularen Verhältnis in der Säure vor.

Es erübrigen sich nunmehr alle Erörterungen, ob die Pyrimidine bei der Hydrolyse aus den Purinen hervorgehen könnten; Fragen, die, schon längst erledigt, immer noch wieder einmal in der Literatur auftauchen. Desgleichen werden die Betrachtungen und Formeln Sch mie deberg s ${ }^{2}$ ) hinfällig, sowohl seine verwickelten Berechnungen der Nucleinsäure selbst wie auch die Aufstellung und Analyse ganz hypothetischer Spaltungsprodukte: Heminucleinsäure, Nucleotin, Nucleotinphosphorsäure, Substanzen, die sich ihrer ganzen Beschreibung nach deutlich genug als Gemenge charakterisieren.

Der eine Teil der Aufgabe, die Zusammensetzung der Nucleinsäure aufzuklären, wäre also gelöst, es bleibt nunmehr noch festzustellen, wie sich der stickstofffreie Anteil verhält, der beim Sieden mit Schwefelsäure Lävulinsäure liefert.

1) Diese Zeitschrift, Bd. XLHI, S. 403.

2) Arch. f. exp. Pathol. u. Pharmak., Bd. XXXVII, S.110; Bd. XLII, S. 57; Bd. LI, S. 239. 